\title{
Zigzag Structure of Thin Chamber Complexes
}

\author{
Michel Deza 1 . Mark Pankov²
}

Received: 12 September 2015 / Revised: 20 October 2017 / Accepted: 7 November 2017 /

Published online: 1 December 2017

(C) The Author(s) 2017. This article is an open access publication

\begin{abstract}
Zigzags in thin chamber complexes are investigated, in particular, all zigzags in the Coxeter complexes are described. Using this description, we show that the lengths of all zigzags in the simplex $\alpha_{n}$, the cross-polytope $\beta_{n}$, the 24-cell, the icosahedron and the 600-cell are equal to the Coxeter numbers of $\mathrm{A}_{n}, \mathrm{~B}_{n}=\mathrm{C}_{n}, \mathrm{~F}_{4}$ and $\mathrm{H}_{i}, i=3,4$, respectively. We also discuss in which cases two faces in a thin chamber complex can be connected by a zigzag.
\end{abstract}

Keywords Zigzag · Thin chamber complex · Coxeter complex

\section{Introduction}

In the present paper, we investigate zigzags in thin chamber complexes which are interesting in the context of Tits buildings [15]. In fact, these are abstract polytopes, where all facets are simplices. The well-known Coxeter complexes form an important subclass of thin chamber complexes. The zigzags in a complex are the orbits of the action of a special operator $T$ on the set of flags of this complex. The operator $T$ transfers every flag $F$ to a flag whose faces are adjacent to the faces of $F$. The main

\footnotetext{
Editor in charge: János Pach

Michel Deza

Michel.Deza@ens.fr

Mark Pankov

pankov@matman.uwm.edu.pl

1 École Normale Supérieure, Paris, France

2 Faculty of Mathematics and Computer Science, University of Warmia and Mazury, Olsztyn, Poland
} 
question under discussion is the following: in which cases can two faces of a complex be connected by a zigzag?

The notion of Petrie polygon for polytopes is one of the central concepts of famous Coxeter's book [2]. For embedded graphs the same objects appear as zigzags in [4,6,7], geodesics in [9] and left-right paths in [14]. Their high-dimensional analogues are considered in [5,16], see also [7, Chap. 8]. Following [4-7] we call such objects zigzags.

Consider an abstract polytope $\mathcal{P}$ of rank $n$ and one of its flags $F$. Let $X_{i}$ be the $i$-face from this flag. There is the unique $i$-face $X_{i}^{\prime}$ adjacent to $X_{i}$ and incident to all other faces from $\mathcal{P}$. We define $\sigma_{i}(F)$ as the flag obtained from $F$ by replacing $X_{i}$ on $X_{i}^{\prime}$ and introduce the operator

$$
T=\sigma_{n-1} \cdots \sigma_{0},
$$

which acts on the set of all flags. In [5,7] zigzags are defined as the orbits of this action. Similarly, for every permutation $\delta$ on the set $\{0,1, \ldots, n-1\}$ we consider the operator

$$
T_{\delta}=\sigma_{\delta(n-1)} \cdots \sigma_{\delta(0)}
$$

and come to generalized zigzags. Note that such objects were first considered in [16] and named Petrie schemes.

Now, let us consider the associated flag complex $\mathfrak{F}(\mathcal{P})$ which is a thin chamber complex of the same rank $n$. In Sect. 3.4, we show that there is a natural one-toone correspondence between generalized zigzags in $\mathcal{P}$ and zigzags in $\mathfrak{F}(\mathcal{P})$. For this reason, it is natural to restrict the general zigzag theory on the case of zigzags in thin chamber complexes and associated with the operator $T$ only.

We describe all zigzags of Coxeter complexes in terms of Coxeter elements and show that the length of zigzags depends on the associated Coxeter number (Sect. 3.3). For example, the Coxeter complexes for $\mathrm{A}_{n}, \mathrm{~B}_{n}=\mathrm{C}_{n}, \mathrm{~F}_{4}$ and $\mathrm{H}_{i}, i=3,4$, are the flag complexes of the simplex $\alpha_{n}$, the cross-polytope $\beta_{n}$, the 24-cell, the icosahedron and the 600-cell (respectively) and all zigzags in these complexes are induced by generalized zigzags in the above mentioned polytopes. In particular, this implies that the lengths of zigzags in the polytopes are equal to the corresponding Coxeter numbers. On the other hand, the Coxeter systems $\mathrm{D}_{n}$ and $\mathrm{E}_{i}, i=6,7,8$, are related to the halfcube $\frac{1}{2} \gamma_{n}$ and the E-polytopes $221,3_{21}, 4_{21}$ (respectively). However, the associated Coxeter numbers are different from the lengths of zigzags in the polytopes. We explain why this occurs.

We say that two faces are z-connected if there is a zigzag joining them. If a thin chamber complex is z-simple, i.e. every zigzag is not self-intersecting, then the $z$ connectedness of any pair of faces implies that the complex is a simplex. The main result of this part concerns the $z$-connectedness of facets (Theorem 4.7). We consider the graph consisting of all facets, whose edges are pairs intersecting in a ridge. We determine a class of path geodesics in this graph which can be extended to zigzags. For $z$-simple complexes, this gives the full description of path geodesics extendible to zigzags. 


\section{Thin Simplicial Complexes}

\subsection{Definitions and Examples}

Let $\Delta$ be an abstract simplicial complex over a finite set $V$, i.e. $\Delta$ is formed by subsets of $V$ such that every one-element subset belongs to $\Delta$ and for every $X \in \Delta$ all subsets of $X$ belong to $\Delta$. Elements of $\Delta$ are called faces and maximal faces are said to be facets. We say that $X \in \Delta$ is a $k$-face if $|X|=k+1$. Recall that 0 -faces and 1 -faces are known as vertices and edges, respectively, and the empty set is the unique (-1)-face. We will always suppose that the simplicial complex $\Delta$ is pure, i.e. all facets are of the same cardinality $n$. Then the number $n$ is the rank of the simplicial complex.

Our second assumption is that $\Delta$ is thin. This means that every ridge, i.e. $(n-2)$ face, is contained in precisely two distinct facets (cf. [15, Sect. 1.3]). Two facets are said to be adjacent if their intersection is a ridge.

Let $k$ be a natural number not greater than $n-2$. If a $(k-1)$-face $Y$ is contained in a $(k+1)$-face $Z$, then there are precisely two $k$-faces $X_{1}$ and $X_{2}$ such that

$$
Y \subset X_{i} \subset Z \text { for } i=1,2
$$

(the set $Z \backslash Y$ contains only two vertices and $X_{1}, X_{2}$ are the $k$-faces containing $Y$ and one of these vertices). We say that two distinct $k$-faces $X_{1}$ and $X_{2}$ are adjacent if there exist a $(k-1)$-face $Y$ and a $(k+1)$-face $Z$ satisfying the above inclusion.

For every $k \in\{0,1, \ldots, n-1\}$ we denote by $\Gamma_{k}(\Delta)$ the graph whose vertex set consists of all $k$-faces and whose edges are pairs of adjacent faces. This graph is one of so-called Wythoff kaleidoscopes [2]. Following [15, Sect. 1.3] we say that $\Delta$ is a chamber complex if the graph $\Gamma_{n-1}(\Delta)$ is connected. This condition guarantees that $\Gamma_{k}(\Delta)$ is connected for every $k$. Indeed, for any two $k$-faces $X, Y$ with $k<n-1$ we take facets $X^{\prime}, Y^{\prime}$ containing $X, Y$ (respectively); using a path in $\Gamma_{n-1}(\Delta)$ connecting $X^{\prime}$ with $Y^{\prime}$ we construct a path of $\Gamma_{k}(\Delta)$ connecting $X$ with $Y$.

Let us consider a connected simple graph. The path distance $d(v, w)$ between vertices $v$ and $w$ in this graph is the smallest number $d$ such that there is a path of length $d$ connecting these vertices (see, for example, [3, Sect. 15.1]). Every path of length $d(v, w)$ connecting $v$ and $w$ is called a geodesic. The path distance on $\Gamma_{k}(\Delta)$ will be considered in Sect. 4.

Example 2.1 The $n$-simplex $\alpha_{n}$ is the simplicial complex whose vertex set is the $(n+$ 1)-element set

$$
[n+1]=\{1, \ldots, n+1\}
$$

and whose non-empty faces are proper subsets of $[n+1]$. The cross-polytope $\beta_{n}$ is the simplicial complex whose vertex set is the set

$$
[n]_{ \pm}=\{1, \ldots, n,-1, \ldots,-n\}
$$


and whose faces are all subsets $X \subset[n]_{ \pm}$such that for every $i \in X$ we have $-i \notin X$. It is clear that $\alpha_{n}$ and $\beta_{n}$ are thin chamber complexes of rank $n$. Every $\Gamma_{k}\left(\alpha_{n}\right)$ is the Johnson graph $J(n+1, k+1)$ and $\Gamma_{n-1}\left(\beta_{n}\right)$ is the $n$-dimensional cube graph.

Example 2.2 Let $\Delta_{1}$ and $\Delta_{2}$ be thin chamber complexes over sets $V_{1}$ and $V_{2}$, respectively. The join $\Delta_{1} * \Delta_{2}$ is the simplicial complex whose vertex set is the disjoint union $V_{1} \sqcup V_{2}$ and whose faces are all subsets of type $X_{1} \sqcup X_{2}$, where $X_{i} \in \Delta_{i}$. This is a thin chamber complex of rank $n_{1}+n_{2}$, where $n_{i}$ is the rank of $\Delta_{i}$.

Example 2.3 Let $\mathcal{P}$ be a partially ordered set presented as the disjoint union of subsets $\mathcal{P}_{-1}, \mathcal{P}_{0}, \ldots, \mathcal{P}_{n-1}, \mathcal{P}_{n}$ such that for any $X \in \mathcal{P}_{i}$ and $Y \in \mathcal{P}_{j}$ satisfying $X<Y$ we have $i<j$. The elements of $\mathcal{P}_{k}$ are called $k$-faces. There is the unique $(-1)$-face and the unique $n$-face which are the minimal and maximal elements, respectively. Also, we suppose that every flag, i.e. a maximal linearly ordered subset, contains precisely $n$ elements distinct from the $(-1)$-face and $n$-face. Then $\mathcal{P}$ is an abstract polytope of rank $n$ if the following conditions hold:

(P1) If $k \in\{0,1, \ldots, n-1\}$, then for any $(k-1)$-face $Y$ and $(k+1)$-face $Z$ satisfying $Y<Z$ there are precisely two $k$-faces $X_{i}, i=1,2$, such that $Y<X_{i}<Z$.

(P2) $\mathcal{P}$ is strongly connected (see, for example, [12]).

Every thin chamber complex of rank $n$ can be considered as an abstract $n$-polytope whose $(n-1)$-faces are $(n-1)$-simplices. If $\mathcal{P}$ is an abstract $n$-polytope, then the associated flag complex $\mathfrak{F}(\mathcal{P})$ is the simplicial complex whose vertices are the faces of $\mathcal{P}$ and whose facets are the flags. This is a thin simplicial complex of rank $n$ and (P2) guarantees that $\mathfrak{F}(\mathcal{P})$ is a chamber complex.

A simplicial complex is $k$-neighborly if any $k$ distinct vertices form a face. Then $\alpha_{n}$ can be characterized as the unique $n$-neighborly thin chamber complex of rank $n$. We will use the following fact which follows from a more general result [8, p. 123].

Fact 2.4 If a thin chamber complex of rank $n$ is $k$-neighborly and $k>\lfloor n / 2\rfloor$, then it is the $n$-simplex $\alpha_{n}$.

Example 2.5 The join $\alpha_{n} * \alpha_{m}$ with $n \leq m$ is a thin chamber complex of rank $n+m$ which is $n$-neighborly and not $(n+1)$-neighborly.

\subsection{Coxeter Complexes}

Let $W$ be a finite group generated by a set $S$ whose elements are involutions and denoted by $s_{1}, \ldots, s_{n}$. For any distinct $i, j \in[n]$ we write $m_{i j}$ for the order of the element $s_{i} s_{j}$. Then $m_{i j}=m_{j i} \geq 2$ and the condition $m_{i j}=2$ is equivalent to the fact that $s_{i}$ and $s_{j}$ commute. We suppose that $(W, S)$ is a Coxeter system, i.e. $W$ is the quotient of the free group over $S$ by the normal subgroup generated by all elements of type $\left(s_{i} s_{j}\right)^{m_{i j}}$. The associated diagram $\mathrm{D}(W, S)$ is the graph whose vertex set is $S$ and $s_{i}$ is connected with $s_{j}$ by an edge of order $m_{i j}-2$ (the vertices are disjoint if $m_{i j}=2$ ). All finite Coxeter systems are known [11], in particular, every finite irreducible Coxeter system is one of the following:

$$
\mathrm{A}_{n}, \mathrm{~B}_{n}=\mathrm{C}_{n}, \mathrm{D}_{n}, \mathrm{~F}_{4}, \mathrm{E}_{i}, i=6,7,8, \mathrm{H}_{i}, i=3,4, \mathrm{I}_{2}(m) .
$$


For every subset $I=\left\{i_{1}, \ldots, i_{k}\right\} \subset[n]$ we denote by $W^{I}$ the subgroup generated by the set

$$
S \backslash\left\{s_{i_{1}}, \ldots, s_{i_{k}}\right\}
$$

In particular, for every $i \in[n]$ the subgroup $W^{i}$ is generated by $S \backslash\left\{s_{i}\right\}$. The following properties are well-known (see, for example, [1, Sect. 2.4]):

(C1) $W^{I} \cap W^{J}=W^{I \cup J}$ for any subsets $I, J \subset[n]$,

(C2) if $v, w \in W$ and $I, J \subset[n]$ then we have $w W^{I}=v W^{J}$ only in the case when $I=J$ and $w^{-1} v \in W^{I}$.

Also, we will use the following obvious equality:

$$
s_{i} W^{j}=W^{j} \text { if } i \neq j \text {. }
$$

The Coxeter complex $\Sigma(W, S)$ is the simplicial complex whose vertices are subsets of type $w W^{i}$ with $w \in W$ and $i \in[n]$. The vertices $X_{1}, \ldots, X_{k}$ form a face if there exists $w \in W$ such that

$$
X_{1}=w W^{i_{1}}, \ldots, X_{k}=w W^{i_{k}}
$$

This face can be identified with the set

$$
X_{1} \cap \cdots \cap X_{k}=w W^{I} \text {, where } I=\left\{i_{1}, \ldots, i_{k}\right\} .
$$

Every facet is of type $\left\{w W^{1}, \ldots, w W^{n}\right\}$ and identified with the element $w$. So, there is a natural one-to-one correspondence between facets of $\Sigma(W, S)$ and elements of the group $W$.

Example 2.6 The Coxeter complexes for $\mathrm{A}_{n}, \mathrm{~B}_{n}=\mathrm{C}_{n}, \mathrm{~F}_{4}$ and $\mathrm{H}_{i}, i=3$, 4, are the flag complexes of $\alpha_{n}, \beta_{n}$, the 24-cell, the icosahedron and the 600-cell, respectively. Note that the 24-cell is not a simplicial complex.

For every $w \in W$ the left multiplication $L_{w}$ sending $v W^{i}$ to $w v W^{i}$ is an automorphism of the complex $\Sigma(W, S)$. Also, automorphisms of the diagram $\mathrm{D}(W, S)$ (if they exist) induce automorphisms of $\Sigma(W, S)$. The automorphism group of $\Sigma(W, S)$ is generated by the left multiplications and the automorphisms induced by automorphisms of the diagram (this statement easily follows from [1, Corollary 3.2.6]).

The graph $\Gamma_{n-1}(\Sigma(W, S))$ coincides with the Cayley graph $\mathrm{C}(W, S)$ whose vertex set is $W$ and elements $w, v \in W$ are adjacent vertices if $v=w s_{i}$ for a certain $s_{i} \in S$. Indeed, maximal faces $\left\{w W^{1}, \ldots, w W^{n}\right\}$ and $\left\{v W^{1}, \ldots, v W^{n}\right\}$ are different precisely in one vertex if and only if there is a unique $i \in[n]$ such that $w W^{i} \neq v W^{i}$, in other words,

$$
w^{-1} v \in \bigcap_{j \neq i} W^{j}=\left\langle s_{i}\right\rangle
$$

and we get the required equality. 
Example 2.7 For the dihedral Coxeter system $\mathrm{I}_{2}(\mathrm{~m})$ the Cayley graph is the $(2 \mathrm{~m})$ cycle. The Cayley graph of $\mathrm{A}_{n}$ is the 1-skeleton of the permutohedron [17]; in the general case, the corresponding polytope is called a $W$-permutohedron [10]. See [1, Figs. 3.3] for the Cayley graph of $\mathrm{H}_{3}$.

The length $l(w)$ of an element $w \in W$ is the smallest length of an expression for $w$ consisting of elements from $S$. Such an expression is called reduced if its length is equal to $l(w)$. Note that elements in a reduced expression are not necessarily mutually distinct. The path distance between $v, w \in W$ in the Cayley graph $\mathrm{C}(W, S)$ is equal to $l\left(v^{-1} w\right)=l\left(w^{-1} v\right)$.

\section{Zigzags in Thin Simplicial Complexes}

In this section, we will always suppose that $\Delta$ is a thin simplicial complex of rank $n$ over a finite set $V$. Sometimes, $\Delta$ is assumed to be a thin chamber complex.

\subsection{Flags}

Every flag of $\Delta$ can be obtained from a certain sequence of $n$ vertices which form a facet. Indeed, if $x_{0}, x_{1}, \ldots, x_{n-1}$ is such a sequence, then the corresponding flag is

$$
\left\{x_{0}\right\} \subset\left\{x_{0}, x_{1}\right\} \subset \cdots \subset\left\{x_{0}, x_{1}, \ldots, x_{n-1}\right\} .
$$

Any reenumeration of these vertices gives another flag which contains the facet consisting of the vertices.

If $F$ is the flag corresponding to a vertex sequence $x_{0}, x_{1}, \ldots, x_{n-1}$, then the flag $R(F)$ obtained from the reverse sequence $x_{n-1}, \ldots, x_{1}, x_{0}$ is called the reverse of $F$, in other words, the reverse of (2) is the flag

$$
\left\{x_{n-1}\right\} \subset\left\{x_{n-1}, x_{n-2}\right\} \subset \cdots \subset\left\{x_{n-1}, \ldots, x_{1}\right\} \subset\left\{x_{n-1}, \ldots, x_{1}, x_{0}\right\} .
$$

This definition is equivalent to [7, Def. 8.2].

Now, let $F$ be the flag formed by $X_{0}, \ldots, X_{n-1}$, where every $X_{i}$ is an $i$-face. Then for every $i \in\{0, \ldots, n-1\}$ there is the unique $i$-face $X_{i}^{\prime}$ adjacent to $X_{i}$ and incident to all other $X_{j}$. We denote by $\sigma_{i}(F)$ the flag obtained from $F$ by replacing $X_{i}$ on $X_{i}^{\prime}$. For every flag $F$ we define

$$
T(F):=\sigma_{n-1} \cdots \sigma_{1} \sigma_{0}(F) .
$$

Suppose that $F$ is the flag obtained from a vertex sequence $x_{0}, x_{1}, \ldots, x_{n-1}$. There is the unique vertex $x_{n} \neq x_{0}$ such that $x_{1}, \ldots, x_{n}$ form a facet. An easy verification shows that $T(F)$ is the flag corresponding to the sequence $x_{1}, \ldots, x_{n}$.

Lemma 3.1 For every flag $F$ we have $T R T(F)=R(F)$. 
Proof As above, we suppose that $x_{0}, x_{1}, \ldots, x_{n-1}$ and $x_{1}, \ldots, x_{n}$ are the sequences corresponding to the flags $F$ and $T(F)$, respectively. Then the sequence $x_{n}, \ldots, x_{1}$ corresponds to the flag $R T(F)$. This implies that $T R T(F)$ is defined by the sequence $x_{n-1}, \ldots, x_{1}, x_{0}$ and we get the claim.

Using similar arguments we can prove the following.

Lemma 3.2 If $A$ is an automorphism of $\Delta$, then for every flag $F$ we have $A T(F)=$ $T A(F)$.

\subsection{Zigzags and Their Shadows}

For every flag $F$ the sequence

$$
Z=\left\{T^{i}(F)\right\}_{i \in \mathbb{N}}
$$

(we assume that 0 belongs to $\mathbb{N}$ ) is called a zigzag. Since our simplicial complex is finite, we have $T^{l}(F)=F$ for some $l>0$. The smallest number $l>0$ satisfying this condition is said to be the length of the zigzag. For any number $i$ the sequence

$$
T^{i}(F), T^{i+1}(F), \ldots
$$

is also a zigzag; it is obtained from $Z$ by a cyclic permutation of the flags. All such zigzags will be identified with $Z$.

The $k$-shadow of a zigzag $\left\{F_{i}\right\}_{i \in \mathbb{N}}$ is the sequence $\left\{X_{i}\right\}_{i \in \mathbb{N}}$, where every $X_{i}$ is the $k$-face from the flag $F_{i}$.

Proposition 3.3 Every zigzag can be uniquely reconstructed from any of the shadows.

Proof Let $Z=\left\{F_{i}\right\}_{i \in \mathbb{N}}$ be a zigzag of length $l$ and let $\left\{X_{i}\right\}_{i \in \mathbb{N}}$ be the $k$-shadow of $Z$. The required statement is a consequence of the following two observations. If $k>0$ then the $(k-1)$-shadow is the sequence

$$
X_{l-1} \cap X_{0}, X_{0} \cap X_{1}, X_{1} \cap X_{2}, \ldots
$$

Similarly, if $k<n-1$ then the $(k+1)$-shadow consists of all $X_{i} \cup X_{i+1}$.

Proposition 3.4 If a sequence $Z=\left\{F_{1}, \ldots, F_{l}\right\}$ is a zigzag of lengthl, then the same holds for the sequence

$$
R(Z)=\left\{R\left(F_{l}\right), R\left(F_{l-1}\right), \ldots, R\left(F_{1}\right)\right\} .
$$

Proof If $Z=\left\{F_{1}, \ldots, F_{l}\right\}$ is a zigzag, then for every $i \in[l]$ we have $F_{i}=T^{i-1}(F)$, where $F=F_{1}$. By Lemma 3.1,

$$
T R\left(F_{i}\right)=T R T^{i-1}(F)=R T^{i-2}(F)=R\left(F_{i-1}\right)
$$


if $i \geq 2$ and

$$
T R\left(F_{1}\right)=T R T^{l}(F)=R T^{l-1}(F)=R\left(F_{l}\right) .
$$

This means that $R(Z)$ is a zigzag of length $l$.

Following [7, Def. 8.2], we say that the zigzag $R(Z)$ is the reverse of $Z$.

Remark 3.5 Let $Z=\left\{F_{1}, \ldots, F_{l}\right\}$ be a zigzag. Denote by $F_{i}^{k}$ the $k$-face belonging to the flag $F_{i}$. For every $k$ the sequence $F_{1}^{k}, \ldots, F_{l}^{k}$ is the $k$-shadow of $Z$. The $(n-1)$ shadow of the reverse zigzag is

$$
F_{l}^{n-1}, F_{l-1}^{n-1}, \ldots, F_{1}^{n-1}
$$

Then, by Proposition 3.3, the $(n-2)$-shadow of the reverse zigzag is

$$
F_{1}^{n-2}, F_{l}^{n-2}, F_{l-1}^{n-2}, \ldots, F_{2}^{n-2} .
$$

Step by step, we establish that

$$
F_{i}^{n-i-1}, F_{i-1}^{n-i-1}, \ldots, F_{1}^{n-i-1}, F_{l}^{n-i-1}, \ldots, F_{i+1}^{n-i-1}
$$

is the $(n-i-1)$-shadow of the reverse zigzag for every $i$ satisfying $1 \leq i \leq n-1$. In particular, if $x_{1}, \ldots, x_{l}$ is the 0 -shadow of $Z$, then

$$
x_{n-1}, x_{n-2}, \ldots, x_{1}, x_{l}, x_{l-1}, \ldots, x_{n}
$$

is the 0-shadow of $R(Z)$.

Consider the flag $F$ defined by a vertex sequence $x_{0}, x_{1}, \ldots, x_{n-1}$, i.e.

$$
\left\{x_{0}\right\} \subset\left\{x_{0}, x_{1}\right\} \subset \cdots \subset\left\{x_{0}, x_{1}, \ldots, x_{n-1}\right\} .
$$

There is the unique facet containing $x_{1}, \ldots, x_{n-1}$ and distinct from the facet of $F$. In this facet, we take the unique vertex $x_{n}$ distinct from $x_{1}, \ldots, x_{n-1}$. It was noted above that $T(F)$ is the flag

$$
\left\{x_{1}\right\} \subset\left\{x_{1}, x_{2}\right\} \subset \cdots \subset\left\{x_{1}, \ldots, x_{n}\right\} .
$$

We apply the same arguments to the latter flag and get a certain vertex $x_{n+1}$. Recurrently, we construct a sequence of vertices $\left\{x_{i}\right\}_{i \in \mathbb{N}}$ such that $T^{i}(F)$ is the flag

$$
\left\{x_{i}\right\} \subset\left\{x_{i}, x_{i+1}\right\} \subset \cdots \subset\left\{x_{i}, \ldots, x_{i+n-1}\right\} .
$$

The sequence $\left\{x_{i}\right\}_{i \in \mathbb{N}}$ is the 0 -shadow of the zigzag $\left\{T^{i}(F)\right\}_{i \in \mathbb{N}}$. For every $i \in \mathbb{N}$ the following assertions are fulfilled: 
(Z1) $x_{i}, x_{i+1}, \ldots, x_{i+n-1}$ form a facet,

(Z2) $x_{i} \neq x_{n+i}$.

If $l$ is the length of the zigzag, then $l>n$ and $x_{i+l}=x_{i}$ for all $i \in \mathbb{N}$. The equality $x_{i}=x_{j}$ is possible for some distinct $i, j \in\{0,1, \ldots, l-1\}$ only in the case when $|i-j|>n$. We say that the zigzag $\left\{T^{i}(F)\right\}_{i \in \mathbb{N}}$ is simple if $x_{0}, x_{1} \ldots, x_{l-1}$ are mutually distinct. In this case, the $k$-shadow is formed by $l$ mutually distinct $k$-faces for every $k$. The complex $\Delta$ is said to be $z$-simple if every zigzag is simple.

Proposition 3.6 If $\left\{x_{i}\right\}_{i \in \mathbb{N}}$ is a sequence of vertices satisfying (Z1) and (Z2) for every $i$, then all flags of type (3) form a zigzag and $\left\{x_{i}\right\}_{i \in \mathbb{N}}$ is the 0 -shadow of this zigzag.

Proof Easy verification.

Suppose that a zigzag $Z$ is the reverse of itself, i.e. $R(Z)$ can be obtained from $Z$ by a cyclic permutation of the flags. Then the 0 -shadow of $Z$ is a sequence of type

$$
x_{0}, x_{1}, \ldots, x_{n-1}, x_{n}, \ldots, x_{m}, \ldots, x_{m}, \ldots, x_{n}, x_{n-1}, \ldots, x_{1}, x_{0}, \ldots
$$

and for a sufficiently large $m$ the distance between two exemplars of $x_{m}$ is not greater than $n$. This contradicts (Z1). So, every zigzag is not the reverse of itself. In what follows, every zigzag will be identified with its reverse.

We say that $\Delta$ is $z$-uniform if all zigzags are of the same length.

Lemma 3.7 If $\Delta$ is z-uniform and the length of zigzags is equal to $l$, then there are precisely $n ! N / 2 l$ zigzags, where $N$ is the number of facets in $\Delta$.

Proof By the definition, zigzags are the orbits of the action of the operator $T$ on the set of flags. Thus, the sum of the lengths of all zigzags is equal to the number of flags. There are precisely $n ! N$ distinct flags. Since every zigzag is identified with its reverse, we get precisely $n ! N / 2 l$ zigzags.

Example 3.8 There is a natural one-to-one correspondence between zigzags of $\alpha_{n}$ and permutation on the set $[n+1]$. Every zigzag is of length $n+1$ and Lemma 3.7 implies that the number of zigzag is equal to $\frac{n !}{2}$.

There is the following characterization of $n$-simplex in terms of the length of zigzags.

Proposition 3.9 Suppose that $\Delta$ is a thin chamber complex. Then it contains a zigzag of length $n+1$ if and only if it is the $n$-simplex $\alpha_{n}$.

Proof By Example 3.8, every zigzag in $\alpha_{n}$ is of length $n+1$. Conversely, suppose that $Z$ is a zigzag of length $n+1$. It follows from (Z1) and (Z2) that the 0 -shadow of $Z$ consists of $n+1$ mutually distinct vertices. Denote by $X$ the subset of $V$ formed by these $n+1$ vertices. By (Z1), every $n$-element subset of $X$ is a facet of $\Delta$. Since every $(n-1)$-element subset of $X$ is the intersection of two such facets, the connectedness of $\Gamma_{n-1}(\Delta)$ guarantees that there are no other facets in $\Delta$. 
Example 3.10 The 0-shadow of every zigzag in $\beta_{n}$ is a sequence of the following type:

$$
i_{1}, \ldots, i_{n},-i_{1}, \ldots,-i_{n},
$$

where $i_{1}, \ldots, i_{n}$ form a facet. Thus, all zigzags are of length $2 n$. Since $\beta_{n}$ has precisely $2^{n}$ facets, Lemma 3.7 shows that there are precisely $2^{n-2}(n-1)$ ! zigzags.

The 0 -shadows of zigzags considered in Examples 3.8 and 3.10 consist of all vertices of the complex. For the general case this fails.

By Lemma 3.2, every automorphism of $\Delta$ sends zigzags to zigzags. We say that $\Delta$ is $z$-transitive if for any two zigzags there is an automorphism of $\Delta$ transferring one of them to the other. The complexes $\alpha_{n}, \beta_{n}$ are $z$-transitive. Also, they are $z$-simple.

\subsection{Zigzags in Coxeter Complexes}

Let $(W, S)$ be, as in Sect. 2.2, a finite Coxeter system and let $s_{1}, \ldots, s_{n}$ be the elements of $S$. For every permutation $\delta$ on the set $[n]$ we consider the corresponding Coxeter element

$$
s_{\delta}:=s_{\delta(1)} \cdots s_{\delta(n)} .
$$

It is well-known that the order of this element does not depend on $\delta$ [11, Sect. 3.16]. This order is denoted by $h$ and called the Coxeter number. All Coxeter numbers are known [11, p. 80, Table 2]. Also, we denote by $E_{\delta}$ the flag in $\Sigma(W, S)$ obtained from the following sequence of vertices

$$
W^{\delta(1)}, \ldots, W^{\delta(n)}
$$

The facet in this flag is identified with the identity element $e$. The second facet containing $W^{\delta(2)}, \ldots, W^{\delta(n)}$ is

$$
\left\{s_{\delta(1)} W^{\delta(2)}=W^{\delta(2)}, \ldots, s_{\delta(1)} W^{\delta(n)}=W^{\delta(n)}, s_{\delta(1)} W^{\delta(1)}\right\} .
$$

This facet is identified with $s_{\delta(1)}$. So, the flag $T\left(E_{\delta}\right)$ is defined by the sequence

$$
W^{\delta(2)}, \ldots, W^{\delta(n)}, s_{\delta(1)} W^{\delta(1)} .
$$

The facet containing $W^{\delta(3)}, \ldots, W^{\delta(n)}, s_{\delta(1)} W^{\delta(1)}$ and distinct from $s_{\delta(1)}$ is

$$
\left\{s_{\delta(1)} s_{\delta(2)} W^{\delta(3)}=W^{\delta(3)}, \ldots, s_{\delta(1)} s_{\delta(2)} W^{\delta(1)}=s_{\delta(1)} W^{\delta(1)}, s_{\delta(1)} s_{\delta(2)} W^{\delta(2)}\right\}
$$

This facet is identified with $s_{\delta(1)} s_{\delta(2)}$ and the flag $T^{2}\left(E_{\delta}\right)$ is related to the vertex sequence

$$
W^{\delta(3)}, \ldots, W^{\delta(n)}, s_{\delta(1)} W^{\delta(1)}, s_{\delta(1)} s_{\delta(2)} W^{\delta(2)}
$$


Similarly, we show that for every $i \in[n-1]$ the flag $T^{i}\left(E_{\delta}\right)$ is defined by the sequence

$$
W^{\delta(i+1)}, \ldots, W^{\delta(n)}, s_{\delta(1)} W^{\delta(1)}, s_{\delta(1)} s_{\delta(2)} W^{\delta(2)}, \ldots, s_{\delta(1)} \cdots s_{\delta(i)} W^{\delta(i)}
$$

and the flag $T^{n}\left(E_{\delta}\right)$ corresponds to the sequence

$$
s_{\delta(1)} W^{\delta(1)}, s_{\delta(1)} s_{\delta(2)} W^{\delta(2)}, \ldots, s_{\delta(1)} \cdots s_{\delta(n)} W^{\delta(n)}
$$

Using (1) we rewrite the latter sequence as follows

$$
s_{\delta} W^{\delta(1)}, \ldots, s_{\delta} W^{\delta(n)} .
$$

Therefore,

$$
T^{n}\left(E_{\delta}\right)=L_{s_{\delta}}\left(E_{\delta}\right)
$$

(recall that $L_{w}$ is the left multiplication sending every $v W^{i}$ to $w v W^{i}$, see Sect. 2.2). Since $L_{s_{\delta}}$ is an automorphism of $\Sigma(W, S)$, Lemma 3.2 implies that

$$
T^{n+i}\left(E_{\delta}\right)=L_{s_{\delta}} T^{i}\left(E_{\delta}\right)
$$

for every $i \in \mathbb{N}$, in particular,

$$
T^{m n}\left(E_{\delta}\right)=L_{s_{\delta}^{m}}\left(E_{\delta}\right) .
$$

Thus the length of the zigzag $\left\{T^{i}\left(E_{\delta}\right)\right\}_{i \in \mathbb{N}}$ is equal to $n h$ and the 0 -shadow is

$$
W^{\delta(1)}, \ldots, W^{\delta(n)}, s_{\delta} W^{\delta(1)}, \ldots, s_{\delta} W^{\delta(n)}, \ldots, s_{\delta}^{h-1} W^{\delta(1)}, \ldots, s_{\delta}^{h-1} W^{\delta(n)} .
$$

The $(n-1)$-shadow of this zigzag is the following:

$$
e, s_{\delta(1)}, s_{\delta(1)} s_{\delta(2)}, \ldots, s_{\delta}, s_{\delta} s_{\delta(1)}, \ldots, s_{\delta}^{2}, s_{\delta}^{2} s_{\delta(1)}, \ldots, s_{\delta}^{h}=e
$$

For every number $m<h$ the element $s_{\delta}^{m}$ does not belong to any $W^{i}$ (see [16, Theorem 3.1]). This implies that the zigzag $\left\{T^{i}\left(E_{\delta}\right)\right\}_{i \in \mathbb{N}}$ is simple.

For every flag $F$ in $\Sigma(W, S)$ there exist $w \in W$ and a permutation $\delta$ on the set $[n]$ such that $F=L_{w}\left(E_{\delta}\right)$. The automorphism $L_{w}$ sends the zigzag $\left\{T^{i}\left(E_{\delta}\right)\right\}_{i \in \mathbb{N}}$ to the zigzag $\left\{T^{i}(F)\right\}_{i \in \mathbb{N}}$. The 0 -shadow of the latter zigzag is

$$
w W^{\delta(1)}, \ldots, w W^{\delta(n)}, \ldots, w s_{\delta}^{h-1} W^{\delta(1)}, \ldots, w s_{\delta}^{h-1} W^{\delta(n)}
$$

and the $(n-1)$-shadow is

$$
w, w s_{\delta(1)}, w s_{\delta(1)} s_{\delta(2)}, \ldots, w s_{\delta}, w s_{\delta} s_{\delta(1)}, \ldots, w s_{\delta}^{2}, w s_{\delta}^{2} s_{\delta(1)}, \ldots, w s_{\delta}^{h}=w
$$


Since $\Sigma(W, S)$ is $z$-uniform and contains precisely $|W|$ facets, we can find the number of zigzags in $\Sigma(W, S)$ using Lemma 3.7.

So, we get the following.

Proposition 3.11 The following assertions are fulfilled:

(1) the Coxeter complex $\Sigma(W, S)$ is z-simple;

(2) there are precisely $|W|(n-1) ! / 2 h$ distinct zigzags in $\Sigma(W, S)$ and the length of every zigzag is equal to $n h$, where $h$ is the Coxeter number corresponding to $(W, S)$ and $n=|S|$;

(3) the 0 -shadow and the $(n-1)$-shadow of every zigzag in $\Sigma(W, S)$ are described by the formulas (4) and (5), respectively.

The existence of an automorphism of $\Sigma(W, S)$ transferring the flag defined by the sequence $W^{1}, \ldots, W^{n}$ to the flag associated to the sequence $W^{\delta(1)}, \ldots, W^{\delta(n)}$ is equivalent to the fact that the permutation $\delta$ induces an automorphism of the diagram $\mathrm{D}(W, S)$. So, $\Sigma(W, S)$ is $z$-transitive only in some special cases when every permutation on the set $[n]$ induces an automorphism of the diagram.

\subsection{Generalized Zigzags}

Let $\delta$ be a permutation on the set $\{0,1, \ldots, n-1\}$. Consider the operator

$$
T_{\delta}:=\sigma_{\delta(n-1)} \cdots \sigma_{\delta(1)} \sigma_{\delta(0)}
$$

on the set of flags in $\Delta$. For every flag $F$ the sequence $\left\{T_{\delta}^{i}(F)\right\}_{i \in \mathbb{N}}$ will be called a $\delta$-zigzag. Also, we say that this is a generalized zigzag. As in Sect. 3.2, we define the length and shadows of generalized zigzags.

Remark 3.12 Let $F$ be a flag in $\Delta$ and let $Z=\left\{F, T(F), \ldots, T^{l-1}(F)\right\}$ be the associated zigzag. The operator $\tilde{T}=\sigma_{0} \sigma_{1} \cdots \sigma_{n-1}$ coincides with $T^{-1}$ and we have

$$
\begin{aligned}
& \tilde{T}(F)=T^{l-1}(F), \\
& \tilde{T}^{2}(F)=T^{2 l-2}(F)=T^{l-2}(F), \\
& \vdots \\
& \tilde{T}^{l-1}(F)=T^{(l-1)^{2}}(F)=T(F) .
\end{aligned}
$$

Therefore, $\left\{F, \tilde{T}(F), \ldots, \tilde{T}^{l-1}(F)\right\}$ is the zigzag reversed to $Z$. Similarly, we show that the generalized zigzags defined by the operator $\sigma_{\delta(0)} \sigma_{\delta(1)} \cdots \sigma_{\delta(n-1)}$ are reversed to the generalized zigzags obtained from $T_{\delta}$.

Let $F$ be a flag of $\Delta$ whose $k$-face is denoted by $X_{k}$ for every $k \in\{0,1, \ldots, n-1\}$. This is a facet in the flag complex $\mathfrak{F}(\Delta)$ and we consider the zigzag $Z$ in $\mathfrak{F}(\Delta)$ defined by the vertex sequence

$$
X_{\delta(0)}, X_{\delta(1)}, \ldots, X_{\delta(n-1)}
$$


where $\delta$ is a certain permutation on the set $\{0,1, \ldots, n-1\}$. Let $\left\{Y_{i}\right\}_{i \in \mathbb{N}}$ be the 0 shadow of this zigzag. Then

$$
Y_{0}=X_{\delta(0)}, Y_{1}=X_{\delta(1)}, \ldots, Y_{n-1}=X_{\delta(n-1)}
$$

and $Y_{n}$ is the $\delta(0)$-face in the flag $\sigma_{\delta(0)}(F)$. Similarly, $Y_{n+1}$ is the $\delta(1)$-face in the flag $\sigma_{\delta(1)} \sigma_{\delta(0)}(F)$. Step by step, we establish that $Y_{n+i}$ is the $\delta(i)$-face in the flag

$$
\sigma_{\delta(i)} \cdots \sigma_{\delta(1)} \sigma_{\delta(0)}(F)
$$

for every $i \in\{0,1, \ldots, n-1\}$. Therefore, $Y_{n}, Y_{n+1}, \ldots, Y_{2 n-1}$ belong to the flag $T_{\delta}(F)$. The same arguments show that $Y_{k n+i}$ is the $\delta(i)$-face in the flag $T_{\delta}^{k}(F)$ for every $k \in \mathbb{N}$ and $i \in\{0,1, \ldots, n-1\}$. In other words, the 0 -shadow of $Z$ is formed by the faces from the $\delta$-zigzag $\left\{T_{\delta}^{i}(F)\right\}_{i \in \mathbb{N}}$, i.e.

$$
\underbrace{Y_{0}, Y_{1}, \ldots, Y_{n-1}}_{F}, \underbrace{Y_{n}, \ldots, Y_{2 n-1}}_{T_{\delta}(F)}, \underbrace{Y_{2 n}, \ldots, Y_{3 n-1}}_{T_{\delta}^{2}(F)}, \ldots
$$

Thus the length of $Z$ is equal to $n l$, where $l$ is the length of $\left\{T_{\delta}^{i}(F)\right\}_{i \in \mathbb{N}}$. It is trivial that $Z$ is simple if and only if $\left\{T_{\delta}^{i}(F)\right\}_{i \in \mathbb{N}}$ is simple. In particular, we have proved the following.

Proposition 3.13 There is a natural one-to-one correspondence between zigzags in $\mathfrak{F}(\Delta)$ and generalized zigzags in $\Delta$. The length of a zigzag in $\mathfrak{F}(\Delta)$ is equal to $n l$, where $l$ is the length of the corresponding generalized zigzag in $\Delta$.

Lemma 3.7 implies the following.

Corollary 3.14 If $\mathfrak{F}(\Delta)$ is z-uniform and the length of generalized zigzags in $\Delta$ is equal to $l$, then there are precisely $(n-1) ! N / 2 l$ generalized zigzags, where $N$ is the number of flags in $\Delta$.

Clearly, generalized zigzags can be defined in abstract polytopes and Proposition 3.13 holds for this case. Recall that the flag complexes of $\alpha_{n}, \beta_{n}$, the 24-cell, the icosahedron and the 600-cell are the Coxeter complexes of $\mathrm{A}_{n}, \mathrm{~B}_{n}=\mathrm{C}_{n}, \mathrm{~F}_{4}$ and $\mathrm{H}_{i}$, $i=3,4$ (respectively). Propositions 3.11 and 3.13 imply the following.

Corollary 3.15 The lengths of generalized zigzags in $\alpha_{n}, \beta_{n}$, the 24-cell, the icosahedron and the 600-cell are equal to the corresponding Coxeter numbers

$$
h\left(A_{n}\right)=n+1, h\left(B_{n}\right)=2 n, h\left(F_{4}\right)=12, h\left(H_{3}\right)=10, h\left(H_{4}\right)=30,
$$

respectively.

Note that all values from Corollary 3.14 were given in $[5,7]$, but the connection with Coxeter numbers is new. 
Now, we consider the half-cube $\frac{1}{2} \gamma_{n}$ and the E-polytopes $2_{21}, 3_{21}, 4_{21}$ associated to the Coxeter systems $\mathrm{D}_{n}$ and $\mathrm{E}_{i}, i=6,7,8$ (respectively). The zigzag lengths of these polytopes are not equal to the corresponding Coxeter numbers. The Coxeter numbers of $\mathrm{E}_{i}, i=6,7,8$, are 12,18, 30 and, by [5,7], the zigzag lengths of the E-polytopes are $18,90,36$. The Coxeter number of $\mathrm{D}_{n}$ is equal to $2(n-1)$ and the zigzag length of $\frac{1}{2} \gamma_{n}$ can be found in [5,7] for $n \leq 13$.

The following example explains this non-coincidence.

Example 3.16 For every $i \in[n-1]$ we denote by $\mathcal{F}_{i}$ the set of all $i$-faces in $\beta_{n}$. Then $\mathcal{F}_{n-1}$ can be presented as the disjoint union of two subsets $\mathcal{F}_{+}$and $\mathcal{F}_{-}$satisfying the following condition: for any $X, Y \in \mathcal{F}_{s}, s \in\{+,-\}$ the number $n-|X \cap Y|$ is even and this number is odd if $X \in \mathcal{F}_{+}$and $Y \in \mathcal{F}_{-}$. Suppose that $(W, S)$ is the Coxeter system of type $D_{n}$ (the corresponding diagram is on the figure below).

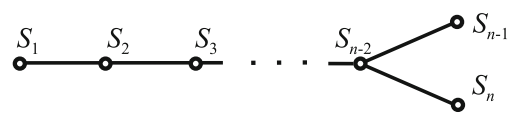

Recall that the vertices of the Coxeter complex $\Sigma(W, S)$ are all subsets $w W^{i}$, $w \in W$ and $i \in[n]$. For every $i \in[n-2]$ the vertices of type $w W^{i}$ correspond to the elements of $\mathcal{F}_{i-1}$ and the vertices of types $w W^{n-1}$ and $w W^{n}$ correspond to the elements of $\mathcal{F}_{+}$and $\mathcal{F}_{-}$(respectively), see [13, Example 2.7] for the details. For every $i \in[n-1]$ the $i$-faces of the hypercube $\gamma_{n}$ can be identified with the elements of $\mathcal{F}_{n-1-i}$. In the case when the vertex set of the half-cube $\frac{1}{2} \gamma_{n}$ is $\mathcal{F}_{+}$, the 2 -faces of $\frac{1}{2} \gamma_{n}$ are the elements of $\mathcal{F}_{n-3} \cup \mathcal{F}_{-}$. In other words, the 2 -faces of $\frac{1}{2} \gamma_{n}$ give two different types of vertices in the Coxeter complex. This means that zigzags in the Coxeter complex of $D_{n}$ cannot be obtained from generalized zigzags in $\frac{1}{2} \gamma_{n}$ as it was described in Proposition 3.13. For the same reason zigzags in the Coxeter complexes of $\mathrm{E}_{i}, i=6,7,8$, are not induced by generalized zigzags of the associated E-polytopes.

\section{Z-Connectedness of Faces}

In this section, we suppose that $\Delta$ is a thin chamber complex of rank $n$. Then every $\Gamma_{k}(\Delta)$ is connected. We say that two faces $X$ and $Y$ in $\Delta$ are $z$-connected if there is a zigzag such that $X$ and $Y$ are contained in some flags of this zigzag.

Example 4.1 In the $n$-simplex $\alpha_{n}$, any two faces are $z$-connected. This easily follows from the fact that every zigzag is defined by a certain enumeration of the set $[n+1]$ (Example 3.8).

Example 4.2 If $n \geq 3$, then the complex $\beta_{n}$ contains pairs of faces which are not $z$-connected. Consider, for example, the edges

$$
\{i, j\} \text { and }\{i,-j\} \text {. }
$$

Up to a cyclic permutation, the 0 -shadow of a zigzag containing $\{i, j\}$ is of type

$$
i, j, i_{1}, \ldots, i_{n-2},-i,-j,-i_{1}, \ldots,-i_{n-2},
$$


where each of $i_{1}, \ldots, i_{n-2}$ is not equal to $\pm i$ or $\pm j$ (see Example 3.10). The corresponding zigzag does not contain $\{i,-j\}$. Similarly, we can show that the $k$-faces

$$
\left\{i_{1}, \ldots, i_{k}, j\right\} \text { and }\left\{i_{1}, \ldots, i_{k},-j\right\}
$$

are not $z$-connected if $k<n-1$. Recall that $\Gamma_{n-1}\left(\beta_{n}\right)$ is the $n$-dimensional cube graph. It is easy to see that every geodesic of this graph is contained in the $(n-1)$-shadow of a certain zigzag.

\subsection{Z-Connectedness of Facets and Normal Geodesics}

If $\Delta$ is $\alpha_{n}$ or $\beta_{n}$, then

$$
d(X, Y)=n-|X \cap Y|
$$

for any two facets $X, Y$. Recall that $d(X, Y)$ is the path distance between $X$ and $Y$ in $\Gamma_{n-1}(\Delta)$. For the general case this distance formula fails. Consider, for example, an $n$-gonal bipyramid; if $n \geq 6$, then it contains two faces $X, Y$ with a common vertex and such that $d(X, Y)>2$. In the general case, we have

$$
d(X, Y) \geq n-|X \cap Y|
$$

for any two facets $X, Y$ in $\Delta$.

We say that facets $X$ and $Y$ form a distance normal pair if the equality (6) holds. In this case, we have $d(X, Y) \leq n$. Any two adjacent facets form a distance normal pair. If the path distance between two facets is equal to 2 , then they form a distance normal pair. It was noted above that any two facets in $\alpha_{n}$ or $\beta_{n}$ form a distance normal pair.

Example 4.3 Let $(W, S)$ be a Coxeter system and $|S|=n$. Consider the associated Coxeter complex $\Sigma(W, S)$. By Sect. 2.2, the graph $\Gamma_{n-1}(\Sigma(W, S))$ can be naturally identified with the Cayley graph $\mathrm{C}(W, S)$. Let $s_{i}$ and $s_{j}$ be non-commuting elements of $S$. The path distance between the identity element $e$ and $w=s_{i} s_{j} s_{i}$ in $\mathrm{C}(W, S)$ is equal to 3 . On the other hand, $e$ and $w$ correspond to the facets $\left\{W^{1}, \ldots, W^{n}\right\}$ and $\left\{w W^{1}, \ldots, w W^{n}\right\}$, respectively. Since we have $w W^{k}=W^{k}$ for every $k \neq i, j$, the intersection of these facets contains precisely $n-2$ vertices and they do not form a distance normal pair.

The latter example can be generalized as follows.

Example 4.4 Let $w$ and $v$ be elements of $W$ such that $l\left(w^{-1} v\right) \leq n$. Then $d(w, v) \leq n$. The facets of $\Sigma(W, S)$ corresponding to $w$ and $v$ form a distance normal pair if and only if there is a reduced expression for $w^{-1} v$ whose elements are mutually distinct (the existence of such an expression implies that all reduced expressions of $w^{-1} v$ satisfy the same condition).

Let $X=X_{0}, X_{1}, \ldots, X_{m}=Y$ be a geodesic in $\Gamma_{n-1}(\Delta)$. Then $d(X, Y)=m$. We say that this geodesic is normal if any two $X_{i}, X_{j}$ satisfying $d\left(X_{i}, X_{j}\right) \leq n$ form a distance normal pair (if $m \leq n$, then this means that $X$ and $Y$ form a distance normal 
pair). If $d(X, Y)>n$, then the fact that $X$ and $Y$ are connected by a normal geodesic does not guarantee that every geodesic connecting them is normal.

Example 4.5 It is not difficult to construct a thin chamber complex of rank 3 satisfying the following conditions:

- there are faces $X, Y$ satisfying $d(X, Y)=4$ and connected by a normal geodesic,

- there is a face $X^{\prime}$ adjacent to $X$ and intersecting $Y$ precisely in a vertex,

- $d\left(X^{\prime}, Y\right)=3$.

So, $X^{\prime}$ and $Y$ do not form a distance normal pair. Let $X^{\prime}, X_{1}, X_{2}, Y$ be a geodesic connecting $X^{\prime}$ and $Y$. The the geodesic $X, X^{\prime}, X_{1}, X_{2}, Y$ is not normal.

The $(n-1)$-shadows of zigzags in $\Delta$ are closed (not necessarily simple) paths in $\Gamma_{n-1}(\Delta)$. We say that a path $X_{1}, \ldots, X_{m}$ is contained in a path $Y_{1}, \ldots, Y_{k}$ (or $Y_{1}, \ldots, Y_{k}$ contains $\left.X_{1}, \ldots, X_{m}\right)$ if there is a number $j$ such that $Y_{j+i}=X_{i}$ for every $i \in\{0,1, \ldots, m\}$. It is clear that every path contained in a normal geodesic is a normal geodesic.

Lemma 4.6 If $Z$ is a simple zigzag in $\Delta$, then every geodesic of $\Gamma_{n-1}(\Delta)$ contained in the $(n-1)$-shadow of $Z$ is normal.

Proof Easy verification.

Theorem 4.7 Every normal geodesic of $\Gamma_{n-1}(\Delta)$ is contained in the $(n-1)$-shadow of a certain zigzag of $\Delta$ and the following assertions are fulfilled:

(1) if this geodesic is of length $m \leq n$, then there are at most $(n-m)$ ! zigzags whose $(n-1)$-shadows contain the geodesic;

(2) if the length of the geodesic is greater than $n$, then it is contained in the $(n-1)$ shadow of the unique zigzag.

Theorem 4.7 together with Lemma 4.6 give the following.

Corollary 4.8 Suppose that $\Delta$ is z-simple. A geodesic of $\Gamma_{n-1}(\Delta)$ is contained in the $(n-1)$-shadow of a certain zigzag if and only if it is normal.

Remark 4.9 Example 4.4 shows that for the Coxeter complexes Theorem 4.7 easily follows from Proposition 3.11.

\subsection{Proof of Theorem 4.7}

Let $X_{0}, X_{1}, \ldots, X_{m}$ be a normal geodesic in $\Gamma_{n-1}(\Delta)$. First, we consider the case when $m \leq n$ and prove the statement by induction.

If $m=1$, then we take vertices $x_{0}, x_{1}, \ldots, x_{n}$ such that

$$
X_{0}=\left\{x_{0}, x_{1}, \ldots, x_{n-1}\right\} \text { and } X_{1}=\left\{x_{1}, \ldots, x_{n}\right\}
$$

For every permutation $\delta$ on the set $[n-1]$ we consider the sequence

$$
x_{0}, x_{\delta(1)}, \ldots, x_{\delta(n-1)}
$$


and denote by $F_{\delta}$ the associated flag

$$
\left\{x_{0}\right\} \subset\left\{x_{0}, x_{\delta(1)}\right\} \subset \cdots \subset\left\{x_{0}, x_{\delta(1)}, \ldots, x_{\delta(n-1)}\right\}=X_{0} .
$$

Since the vertices $x_{\delta(1)}, \ldots, x_{\delta(n-1)}$ belong to $X_{1}$, the facet of the flag $T\left(F_{\delta}\right)$ is $X_{1}$. It is easy to see that every zigzag whose $(n-1)$-shadow contains the path $X_{0}, X_{1}$ is of type $\left\{T^{i}\left(F_{\delta}\right)\right\}_{i \in \mathbb{N}}$. Since $\Delta$ is not assumed to be $z$-simple, the zigzags corresponding to the distinct flags $F_{\delta}$ and $F_{\gamma}$ may be coincident or the zigzag defined by $F_{\delta}$ is the reverse of the zigzag defined by $F_{\gamma}$. Therefore, there are at most $(n-1)$ ! zigzags satisfying the required condition.

Let $m>1$. Then $X_{0}, X_{1}, \ldots X_{m-1}$ is a normal geodesic and, by the inductive hypothesis, it is contained in the $(n-1)$-shadow of a certain zigzag $Z$. Let $\left\{x_{i}\right\}_{i \in \mathbb{N}}$ be the 0 -shadow of $Z$. We suppose that the vertices $x_{0}, x_{1}, \ldots, x_{n-1}$ belong to $X_{0}$. Then

$$
X_{i}=\left\{x_{i}, \ldots, x_{i+n-1}\right\}
$$

for every $i \in[m-1]$.

If $n=m$, then $X_{0} \cap X_{m}=\emptyset$, in particular, $x_{n-1} \notin X_{n}$. Since

$$
X_{n-1}=\left\{x_{n-1}, \ldots, x_{2 n-2}\right\}
$$

and $X_{n}$ are adjacent, the vertices $x_{n}, \ldots, x_{2 n-2}$ belong to $X_{n}$. This implies that $X_{n}$ coincides with the facet $\left\{x_{n}, \ldots, x_{2 n-1}\right\}$ and the geodesic $X_{0}, X_{1}, \ldots, X_{n}$ is contained in the $(n-1)$-shadow of $Z$. This is the unique zigzag whose $(n-1)$-shadow contains this geodesic. Indeed, every $x_{i}, i \in\{0,1, \ldots, n-1\}$, is the unique vertex belonging to $X_{i} \backslash X_{i+1}$ and $Z$ is completely determined by the list of the first $n$ vertices in the 0 -shadow.

Consider the case when $m<n$. The face

$$
A=\left\{x_{m-1}, \ldots, x_{n-1}\right\}
$$

consists of $n-m+1 \geq 2$ vertices and coincides with the intersection of $X_{0}$ and $X_{m-1}$. Since $X_{0}$ and $X_{m}$ form a distance normal pair and $d\left(X_{0}, X_{m}\right)=m$, the face $A \cap X_{m}$ contains precisely $n-m$ vertices. So, there is the unique number $t \in\{m-1, \ldots, n-1\}$ such that $x_{t} \notin X_{m}$. For every permutation $\delta$ on the set $\{m-1, \ldots, n-1\} \backslash t$ we consider the vertex sequence

$$
x_{0}, x_{1}, \ldots, x_{m-2}, x_{t}, x_{\delta(m-1)}, \ldots, \widehat{x_{t}}, \ldots, x_{\delta(n-1)}
$$

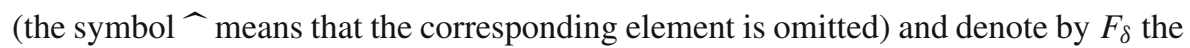
flag obtained from this sequence. This flag defines the zigzag

$$
Z_{\delta}=\left\{T^{i}\left(F_{\delta}\right)\right\}_{i \in \mathbb{N}} \cdot
$$

Obviously, the first $n$ vertices in the 0 -shadow of $Z_{\delta}$ are (7). The next $m-1$ vertices are $x_{n}, \ldots, x_{n+m-2}$ which means that the $(n-1)$-shadow of $Z_{\delta}$ contains the geodesic 
$X_{0}, X_{1}, \ldots, X_{m-1}$. The $m$-th element in the $(n-1)$-shadow of $Z_{\delta}$ is adjacent to $X_{m-1}$ and does not contain $x_{t}$. This implies that it coincides with $X_{m}$. Therefore, the geodesic $X_{0}, X_{1}, \ldots, X_{m}$ is contained in the $(n-1)$-shadow of $Z_{\delta}$. Using the fact that $x_{i}$ is the unique vertex in $X_{i} \backslash X_{i+1}$ for $i \in\{0,1, \ldots, m-2\}, x_{t}$ is the unique vertex in $X_{m-1} \backslash X_{m}$ and

$$
X_{0} \cap X_{m}=X_{0} \cap X_{1} \cap \cdots \cap X_{m}=A \backslash\left\{x_{t}\right\}
$$

we show that every zigzag whose $(n-1)$-shadow contains $X_{0}, X_{1}, \ldots, X_{m}$ is of type $Z_{\delta}$. As above, for distinct permutations $\delta$ and $\gamma$ the zigzags $Z_{\delta}$ and $Z_{\gamma}$ may be coincident. For this reason, there are at most $(n-m)$ ! such zigzags.

Now, we suppose that $m>n$. For every $i \in\{0,1, \ldots, m-n\}$ we have

$$
d\left(X_{i}, X_{i+n}\right)=n
$$

and the facets $X_{i}, X_{i+n}$ form a distance normal pair. It was established above that there is the unique zigzag $Z_{i}$ whose $(n-1)$-shadow contains the geodesic $X_{i}, \ldots, X_{i+n}$. If $i<m-n$, then the geodesic $X_{i+1}, \ldots, X_{i+n}$ is contained in the $(n-1)$-shadows of $Z_{i}$ and $Z_{i+1}$. Since

$$
d\left(X_{i+1}, X_{i+n}\right)=n-1,
$$

there is the unique zigzag whose $(n-1)$-shadow contains $X_{i+1}, \ldots, X_{i+n}$. Thus $Z_{i}$ coincides with $Z_{i+1}$ for every $i<n-m$. This means that all zigzags $Z_{i}$ are coincident and we get the claim.

\subsection{Z-Connectedness of Non-maximal Faces}

Two faces $X$ and $Y$ of the same non-maximal rank $k \geq 1$ are said to be weakly adjacent if their intersection is a $(k-1)$-face and there is no face containing both $X$ and $Y$. This is a modification of the relation defined for non-maximal singular subspaces of polar spaces [13, Subsection 4.6.2]. Note that the non-maximal faces of $\beta_{n}$ considered in Example 4.2 are weakly adjacent.

Lemma 4.10 For every $k \in[n-2]$ any pair of weakly adjacent $k$-faces in $\Delta$ cannot be connected by a simple zigzag.

Proof Let $\left\{x_{i}\right\}_{i \in \mathbb{N}}$ be the 0 -shadow of a zigzag connecting weakly adjacent $k$-faces $X$ and $Y$. If the zigzag is simple, then there exists $i$ such that

$$
X \cap Y=\left\{x_{i+1}, \ldots, x_{i+k}\right\},
$$

one of $X, Y$ is $\left\{x_{i}, \ldots, x_{i+k}\right\}$ and the other is $\left\{x_{i+1}, \ldots, x_{i+k+1}\right\}$. Since the rank $k$ is not maximal, we have $k+2 \leq n$ and

$$
X \cup Y=\left\{x_{i}, \ldots, x_{i+k+1}\right\}
$$


is a face which contradicts the fact that $X$ and $Y$ are weakly adjacent.

A possible $z$-connectedness for two weakly adjacent faces is described in the following example.

Example 4.11 Let $\left\{x_{i}\right\}_{i \in \mathbb{N}}$ be the 0 -shadow of a zigzag. The length $l$ of the zigzag is assumed to be sufficiently large. Also, we suppose that the zigzag is not simple and there exist $i, j \in[l-1]$ and $k<n-1$ such that

$$
i+k \leq j, \quad j+k \leq l-1
$$

and

$$
x_{i}=x_{j}, \quad x_{i+1}=x_{j+1}, \ldots, x_{i+k-1}=x_{j+k-1} .
$$

If the vertices $x_{i-1}, x_{i}, \ldots, x_{i+k-1}, x_{j+k}$ do not form a face (this means that $j+k-i>$ $n-1)$, then

$$
\left\{x_{i-1}, x_{i}, \ldots, x_{i+k-1}\right\} \text { and }\left\{x_{j}, \ldots, x_{j+k-1}, x_{j+k}\right\}
$$

are weakly adjacent $k$-faces connected by our zigzag.

Lemma 4.12 If $\Delta$ is z-simple and any two edges of $\Delta$ are z-connected, then $\Delta$ is 3-neighborly.

Proof By Lemma 4.10, there exist no pairs of weakly adjacent edges, i.e. any two edges with a common vertex are adjacent. If $x_{0}, x_{1}, \ldots, x_{m}$ is a path in $\Gamma_{0}(\Delta)$, then the edges $x_{0} x_{1}$ and $x_{1} x_{2}$ are adjacent which implies that $x_{0}, x_{2}$ are adjacent vertices and $x_{0}, x_{2}, \ldots, x_{m}$ is a path in $\Gamma_{0}(\Delta)$. Step by step, we show that the vertices $x_{0}$ and $x_{m}$ are adjacent. Since the graph $\Gamma_{0}(\Delta)$ is connected, any two distinct vertices are adjacent. Let $x_{1}, x_{2}, x_{3}$ be three distinct vertices of $\Delta$. It was established above that they are mutually adjacent. Then the edges $x_{1} x_{2}$ and $x_{2} x_{3}$ are adjacent and we get the claim.

The previous lemma can be generalized as follows.

Proposition 4.13 If $\Delta$ is z-simple and there is a non-zero number $k<n-1$ such that any two faces of the same non-zero rank $\leq k$ are z-connected, then $\Delta$ is $(k+2)$ neighborly.

Proof The statement coincides with Lemma 4.12 if $k=1$. Let $k \geq 2$. Lemma 4.12 states that $\Delta$ is 3-neighborly. It follows from Lemma 4.10 that for every $i \in[k]$ two $i$-faces are adjacent if their intersection is a $(i-1)$-face. Therefore, if $X$ is a 4-element subset in the vertex set, then any two distinct 3-element subsets of $X$ are adjacent 2 -faces and $X$ is a 3-face. Step by step, we establish that every subset consisting of not greater than $k+2$ vertices is a face.

Proposition 4.13 together with Fact 2.4 give the following.

Corollary 4.14 Suppose that, as in Proposition 4.13, $\Delta$ is z-simple and there is a non-zero number $k<n-1$ such that any two faces of the same non-zero dimension $\leq k$ are $z$-connected. If $k>\lfloor n / 2\rfloor-2$, then $\Delta$ is the $n$-simplex. 
Acknowledgements The authors thank Mathieu Dutour Sikirić for interest and discussion and Christophe Hohlweg for remarks concerning Coxeter groups. Also, they are grateful to anonymous referee who appealed their attention to [16].

Open Access This article is distributed under the terms of the Creative Commons Attribution 4.0 International License (http://creativecommons.org/licenses/by/4.0/), which permits unrestricted use, distribution, and reproduction in any medium, provided you give appropriate credit to the original author(s) and the source, provide a link to the Creative Commons license, and indicate if changes were made.

\section{References}

1. Björner, A., Brenti, F.: Combinatorics of Coxeter Groups. Graduate Texts in Mathematics, vol. 231. Springer, New York (2005)

2. Coxeter, H.S.M.: Regular Polytopes, 3rd edn. Dover, New York (1973)

3. Deza, M.M., Deza, E.: Encyclopedia of Distances, 4th edn. Springer, Berlin (2016)

4. Deza, M., Dutour, M.: Zigzag structure of simple bifaced polyhedra. Comb. Probab. Comput. 14(1-2), 31-57 (2005). arXiv:math/0212352 (2002)

5. Deza, M., Dutour, M.: Zigzag structure of complexes. Southeast Asian Bull. Math. 29(2), 301-320 (2005). arXiv:math/0405279 (2004)

6. Deza, M., Dutour, M., Fowler, P.W.: Zigzags, railroads, and knots in fullerenes. J. Chem. Inf. Comput. Sci. 44(4), 1282-1293 (2004)

7. Deza, M.-M., Dutour Sikirić, M., Shtogrin, M.I.: Geometric Structure of Chemistry-Relevant Graphs: Zigzags and Central Circuits. Forum for Interdisciplinary Mathematics, vol. 1. Springer, New Delhi (2015)

8. Grünbaum, B.: Convex polytopes. In: Kaibel, V., Klee, V., Ziegler, G. (eds.) Graduate Texts in Mathematics, vol. 221, 2nd edn. Springer, New York (2003)

9. Grünbaum, B., Motzkin, T.S.: The number of hexagons and the simplicity of geodesics on certain polyhedra. Can. J. Math. 15, 744-751 (1963)

10. Hohlweg, C.: Permutahedra and associahedra: generalized associahedra from the geometry of finite reflection groups. In: Müller-Hoissen, F., et al. (eds.) Associahedra, Tamari Lattices and Related Structures, Progress in Mathematics, vol. 299, pp. 129-159. Birkhäuser, Basel (2012)

11. Humphreys, J.E.: Reflection Groups and Coxeter Groups. Cambridge Studies in Advanced Mathematics, vol. 29. Cambridge University Press, Cambridge (1990)

12. McMullen, P., Schulte, E.: Abstract Regular Polytopes. Encyclopedia of Mathematics and its Applications, vol. 92. Cambridge University Press, Cambridge (2002)

13. Pankov, M.: Grassmannians of Classical Buildings. Algebra and Discrete Mathematics, vol. 2. World Scientific, Hackensack (2010)

14. Shank, H.: The theory of left-right paths. In: Street, A.P., Wallis, W.D. (eds.) Combinatorial Mathematics III. Lecture Notes in Mathematics, vol. 452, pp. 42-54. Springer, Berlin (1975)

15. Tits, J.: Buildings of Spherical Type and Finite BN-Pairs. Lecture Notes in Mathematics, vol. 386. Springer, Berlin (1974)

16. Williams, G.: Petrie schemes. Can. J. Math. 57(4), 844-870 (2005)

17. Ziegler, G.M.: Lectures on Polytopes. Graduate Texts in Mathematics, vol. 152. Springer, New York (1995) 